\title{
Inducible gene expression system by 3-hydroxypropionic acid
}

\author{
Shengfang Zhou ${ }^{1,2 \dagger}$, Satish Kumar Ainala ${ }^{1 \dagger}$, Eunhee Seol ${ }^{1}$, Trinh Thi Nguyen ${ }^{1}$ and Sunghoon Park ${ }^{1 *}$
}

\begin{abstract}
Background: 3-Hydroxypropionic acid (3-HP) is an important platform chemical that boasts a variety of industrial applications. Gene expression systems inducible by $3-\mathrm{HP}$, if available, are of great utility for optimization of the pathways of 3-HP production and excretion.

Results: Here we report the presence of unique inducible gene expression systems in Pseudomonas denitrificans and other microorganisms. In P. denitrificans, transcription of three genes ( $h p d H, m m s A$ and $h b d H-4$ ) involved in 3-HP degradation was upregulated by 3 -HP by the action of a transcriptional regulator protein, LysR, and a cis-acting regulatory site for LysR binding. Similar inducible systems having an LysR transcriptional regulator were identified in other microorganisms that also could degrade 3-HP. A docking study showed that the 3-HP binding pocket is located between the N-terminal helix-turn-helix motif and the C-terminal cofactor-binding domain.
\end{abstract}

Conclusions: This LysR-regulated 3-HP-inducible system should prove useful for control of the level of gene expression in response to $3-\mathrm{HP}$.

Keywords: 3-Hydroxypropionic acid, LysR, Inducible, hpdH, mmsA, hbdH

\section{Background}

3-Hydroxypropionic acid (3-HP) is an important platform chemical. It can be converted to acrylic acid, acrylamide, 1,3-propanediol, malonic acid, and others. It can also be used for synthesis of biodegradable polymer [1-3]. Biological production of 3-HP from glycerol has been successfully demonstrated in several bacteria such as Escherichia coli, Klebsiella pneumoniae and Pseudomonas denitrificans, which are engineered to (over)express glycerol dehydratase (GDHt, coenzyme $\mathrm{B}_{12}$-dependent), glycerol dehydratase reactivation factors $(\mathrm{Gdr} A B)$, and/or aldehyde dehydrogenase (ALDH, $\mathrm{NAD}^{+}$-dependent) [4-8]. Some recombinant strains such as $E$. coli W DUBGK have produced $>40 \mathrm{~g} / \mathrm{L}$ of $3-\mathrm{HP}$ in $48 \mathrm{~h}$ [9], but further increases of 3-HP production have been challenging. The 3-HP pathway enzymes GDHt and

\footnotetext{
*Correspondence: parksh@pusan.ac.kr

†Shengfang Zhou and Satish Kumar Ainala are co-first authors

1 School of Chemical and Biomolecular Engineering, Pusan National

University, San 30 Jangeon-dong, Geumjeong-gu, Busan 609-735,

Republic of Korea

Full list of author information is available at the end of the article
}

ALDH have been shown to be unstable and to lose their activities during prolonged fermentation, especially in the late stage [9].

One important mechanism of GDHt activity loss is socalled suicidal inactivation. Coenzyme $\mathrm{B}_{12}$, the cofactor of GDHt, is irreversibly damaged during dehydration reaction of the substrate glycerol to 3-hydroxypropionaldehyde (3-HPA) [10]. This inactivation is aggravated in the presence of oxygen. To alleviate this mechanism-based inactivation, Yamanishi et al. [11] developed mutant GDHt by site-directed mutagenesis. Some of the mutant enzymes exhibited improved stability, but the activity was significantly lower than that of the wild type. Toxic intermediate 3-HPA also causes enzyme instability. It was observed that, when GDHt or ALDH was incubated with 3-HPA, its activity declined in a dose-dependent manner (unpublished data). Aldehyde is known to react with amino acid residues lysine, cysteine and histidine by targeting the $\varepsilon$-amino group $\left(\mathrm{NH}_{3}{ }^{+}\right)$, the sulfhydryl group $(-\mathrm{SH})$ and the imidazole group, respectively [12-15]. Serious efforts to stabilize enzymes against aldehydes by 
site-directed or random mutagenesis have been made, but with only limited success $[13,16]$.

One alternative approach to solve the instability problem in 3-HP production is to synthesize new and active enzymes continuously during the entire period of cultivation. This is difficult, especially in the late period of fermentation, where cell growth is very slow and target products often accumulate at levels toxic enough to interfere with cellular metabolisms. However, if there exists a dynamic 3-HP-inducible promoter, gene expression for the aforementioned, unstable GDHt and ALDH enzymes can be stimulated and their enzymatic activities can be maintained at high levels, even in the late period of 3-HP production. To this end, we attempted to screen such promoters in 3-HP-degrading P. denitrificans, and identified two of them in this specific strain. After analyzing the 3-HP-inducible system's gene arrangement, including transcriptional activator proteins, promoter sequences and structural genes under the control of such 3-HP-inducible promoters, similar 3-HP-inducible gene expression systems were searched and identified in other microorganisms. The existence of 3-HP-inducible gene expression systems in many of these microbes was confirmed by measurement of the transcriptions of the genes under the control of the 3-HP-inducible promoters.

\section{Results and discussion}

\section{Screening of 3-HP-inducible promoters in $P$. denitrificans}

3-Hydroxypropionic acid (3-HP) is a carbon compound not commonly encountered in the natural environment; neither its use as a carbon substrate nor its biological degradation has been adequately elucidated. Recently, we found that $P$. denitrificans can grow on 3-HP as carbon source and, further, that it degrades 3-HP under non-growing conditions in the presence of oxygen $[7,8]$. Genome sequence and metabolite analysis by gas chromatography/mass spectrometry [7] suggested that 3-HP is metabolized in $P$. denitrificans sequentially via two major enzymes, a putative 3-hydroxypropionate dehydrogenase $(\mathrm{HpdH})$ and (methyl)malonate-semialdehyde dehydrogenase (MmsA) $[8,17,18]$, and converted to acetyl-CoA. In activity assay with purified enzymes, a putative 3-hydroxyisobutyrate dehydrogenase ( $\mathrm{HbdH}-4)$ also exhibited 3-HP degradation [19]. Because the degradation activity of 3-HP in P. denitrificans was found to increase greatly when cells were exposed to 3-HP [8], we hypothesized that the expressions of these enzymes are induced by 3-HP. Thus, transcription of three chosen 3-HP catabolic genes, $h p d H, h b d H-4$ and mmsA, was examined by quantitative RT-PCR with and without exposure to 3-HP (Fig. 1). The housekeeping gene rpoD, encoding sigma factor 70 , was used as a reference. As expected, expression of all three putative 3-HP catabolic genes was enhanced markedly upon exposure to 3-HP: 47-fold for $h p d H, 141$-fold for $h b d H-4$, and 142-fold for $m m s A$. This indicates that transcription of the three genes is induced by 3-HP. The levels of transcription for $m m s A$ and $h b d H-4$ were much higher than that for $h p d H$, suggesting that the promoter strength of the first two genes is much stronger than the other.

\section{Analysis of 3-HP-inducible gene expression system}

To unravel the details of the 3-HP-induced gene expression systems, the genetic arrangement of the operons for $h p d H, h b d H-4$ and mmsA in P. denitrificans was analyzed (Fig. 2). The three genes were found to exist in two separate operons, $h p d H$ in one operon (the C3 operon, hereafter) and $h b d H-4$ and $m m s A$ in another (the $\mathrm{C} 4$ operon). Located just before each of the $\mathrm{C} 3$ and $\mathrm{C} 4$ operons, there was an oppositely oriented gene encoding a LysR-type transcriptional regulator (LTTR) (Fig. 2a), which suggests the possible involvement of LysR protein in the regulation of the expressions of those genes. Additionally, this suggests that the transcription of LysR regulator protein is also self-regulated, though negatively. LysR protein is known to be composed of a DNA-binding domain (helix-turn-helix motif) at the $\mathrm{N}$-terminus and a cofactor-binding domain at the C-terminus [20]. When binding to DNA, LysR protein forms a homodimer and targets two specific binding sites, a regulatory binding site, which has the conserved T- $\mathrm{N}_{11}-\mathrm{A} / \mathrm{TTA}-\mathrm{N}_{7 / 8}$-GAA motif, and an activation binding site (near the -35 RNA polymerase binding site) (Fig. 2b). The tetramer of LysR, formed through protein-protein interaction between two LysR homodimers, is known to lead to conformational changes in DNA and, thereby, to enhancement of RNA polymerase binding in the promoter sequence. The conserved T- $\mathrm{N}_{11}$-A/TTA- $\mathrm{N}_{7 / 8}$-GAA motif also was identified in both operons. The structure of the putative LysR protein for the $\mathrm{C} 4$ operon was analyzed as well (see Fig. 3 for more details). Features similar to those found in other LysR proteins, namely a DNA-binding helix-turn-helix motif, a substrate-binding motif and a linker connecting the two, were identified in C4-LysR. The structural characteristics of the putative LysR, the existence of the LysRbinding $\mathrm{T}-\mathrm{N}_{11}$-A/TTA- $\mathrm{N}_{7 / 8}$-GAA motif DNA sequence in the intergenic region, as well as the RT-PCR results shown in Fig. 1, strongly support the conclusion that gene transcription in the $\mathrm{C} 3$ and $\mathrm{C} 4$ operons is induced by the LysR-3-HP complex.

\section{Virtual screening of 3-HP-inducible gene expression systems}

The LTTR-mediated 3-HP-inducible gene expression systems were screened in other microorganisms using homology searches of various public and private 

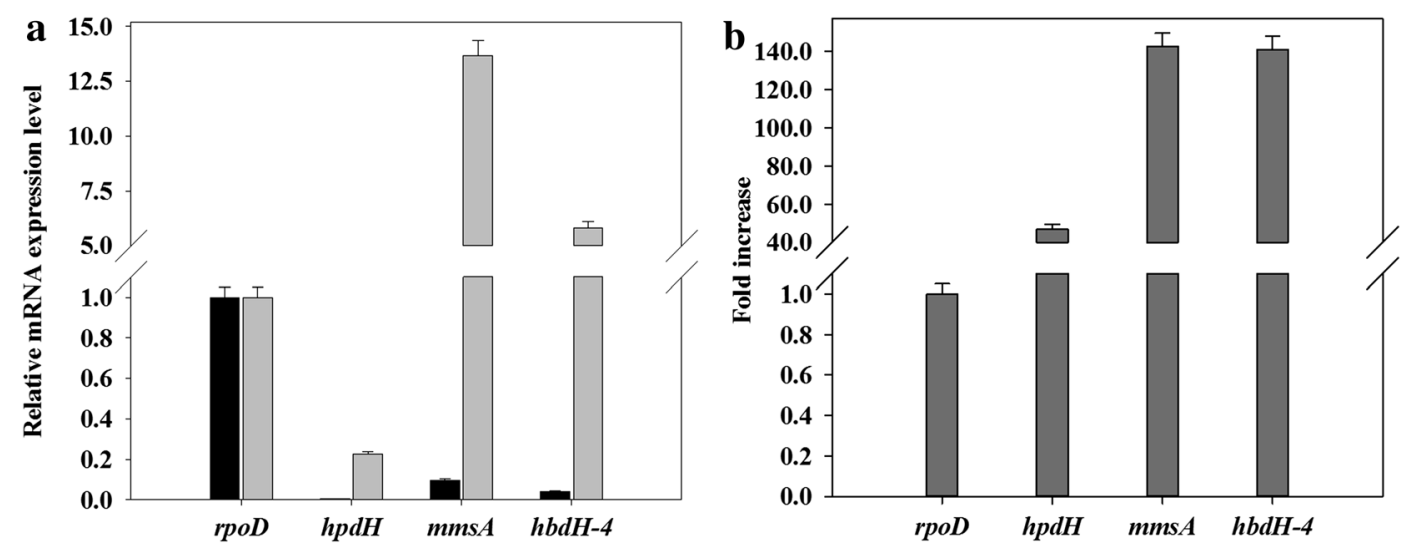

Fig. 1 a Relative mRNA levels and $\mathbf{b}$ fold increase of 3-hydroxypropionate catabolism genes in wild-type P. denitrificans ATCC13867. P. denitrificans cells were cultivated in $\mathrm{M} 9$ minimal medium containing $5 \mathrm{~g} / \mathrm{L}$ sodium gluconate. At $\mathrm{OD}_{600}$ of $\sim 0.4-0.5,3-\mathrm{HP}$ was supplemented at $25 \mathrm{mmol} / \mathrm{L}$ (gray bar) or $0 \mathrm{mM}$ (black bar), and cells were harvested after a further $2 \mathrm{~h}$ cultivation. The fold increase is indicated by the dark gray bar. The standard deviation of the mRNA-level measurements was $<10 \%$. The mRNA levels were compared with those of the reference gene, rpoD

a LysR family transcriptional regulator $(C 3-l y s R)$
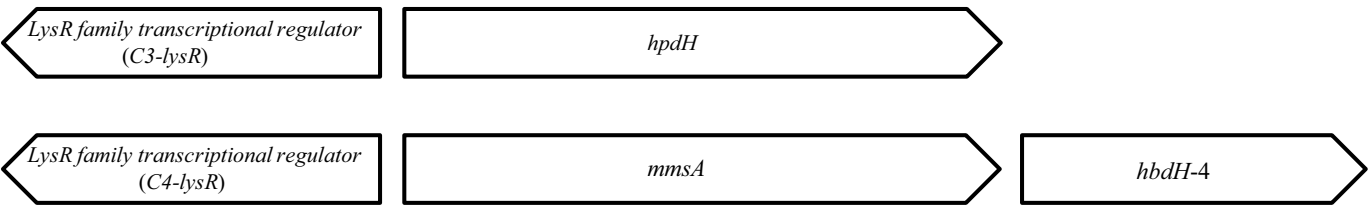

b

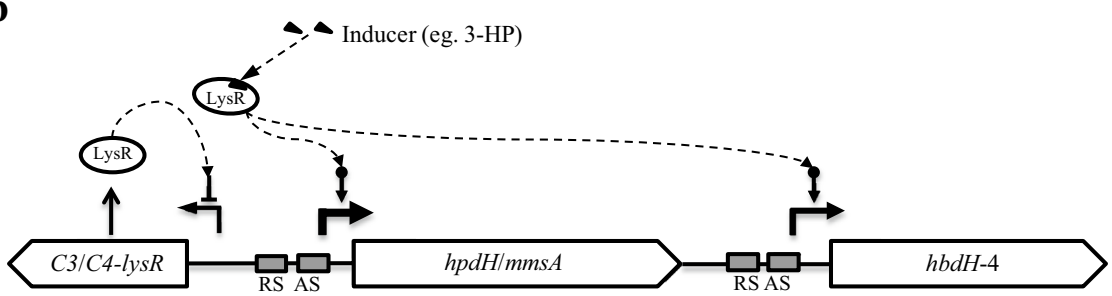

Fig. 2 Genetic arrangement of 3-HP catabolism genes in P. denitrificans and analysis of LysR regulation system. a 3-HP catabolic genes ( $h p d H$, $h b d H-4$ and $m m s A$ ) divergently located relative to lysR-family transcription regulatory gene. $\mathbf{b}$ Hypothetical scheme of typical structure of divergent promoter regulated by LysR-family transcriptional regulator. $R S$ regulatory site, $A S$ activation site, Conserved motifT- $\mathrm{N}_{11}-\mathrm{A}$ or TTA-N $\mathrm{N}_{7 / 8}-\mathrm{GAA}$

databases such as NCBI (nr, refseq_protein, and env_nr), MBGD (Microbial Genome Database), and SEED. The most conserved components of the $\mathrm{C} 3$ and $\mathrm{C} 4$ operons from P. denitrificans, $\mathrm{HpdH}$ (C3-LysR) and $\mathrm{HbdH}-4$ (C4-LysR), were used as primary queries. The 3-HPinducible systems existed in more than 53 genera among the members of the sequenced bacterial species; some of them had both $\mathrm{C} 3$ and $\mathrm{C} 4$ operons, while others $\mathrm{C} 4$ operon only. The 53 genera were arbitrarily grouped into 14 based on (1) their gene organization and (2) the existence of the $\mathrm{C} 3$ operon (Fig. 4). A comparison among the various organisms revealed that the genetic organizations and compositions of the LysR systems differed significantly. Notably for example, the C4 system was more commonly present than the $\mathrm{C} 3$. Interestingly too, in many microorganisms, the genes encoding a DNAbinding regulator protein (C3- or C4-LysR) were located just before the corresponding $\mathrm{C} 3$ or $\mathrm{C} 4$ operon and oppositely oriented, indicating that transcription of the LysR regulator protein is self-repressed, as in the case of $P$. denitrificans (see Fig. 2). However, in other microorganisms, the gene encoding LysR was located remotely from the $\mathrm{C} 3$ or $\mathrm{C} 4$ operon and in the same orientation. Further studies are required to understand the significance of the different LysR gene arrangements among various genera. 


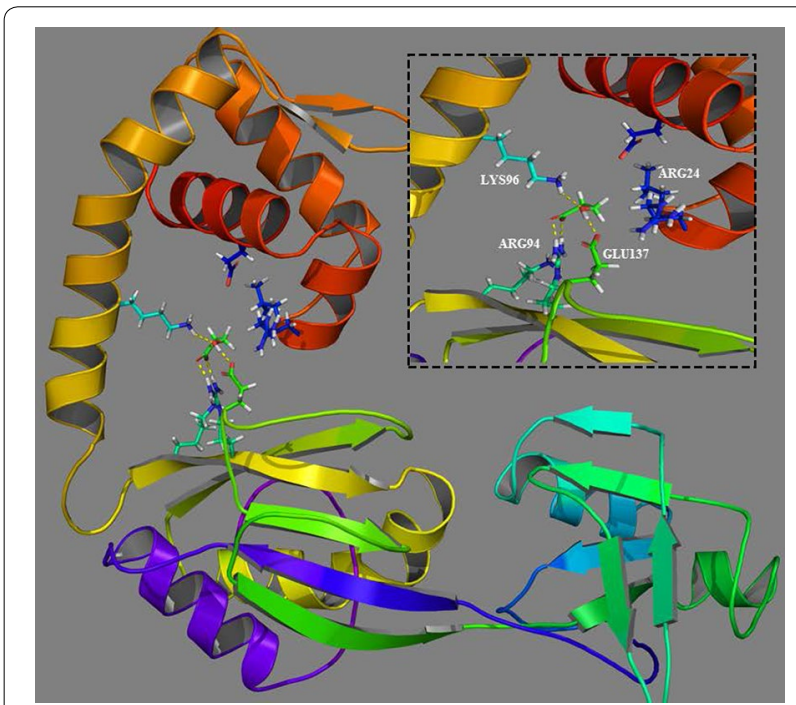

Fig. 3 Predicted three-dimensional (3D) structure and docking analysis of C4-LysR in Pseudomonas denitrificans ATCC 13867. The inset shows the active-site pocket amino acid interactions with 3-HP. The hydrogen bonds are represented by the yellow dotted lines

A phylogenetic tree was generated to analyze the proximities among the C4-LysR variants (Fig. 5). The C4-LysR of Pseudomonas was close to that of Azotobacter but far from those of Kitasatospora, Kutzneria, Segniliparus, Catenulispora and Gordonia. The multiple sequence alignment for the helix-turn-helix regions of C3- and C4-LysR exhibited a high amino acid sequence homology (data not shown). Moreover, the amino acid sequences of the enzymes $\mathrm{HpdH}, \mathrm{HbdH}$ and MmsA appeared to be significantly conserved across all of the species studied (Additional files 1 and 2: Tables S1 and S2). This indicates that the 3-HP-inducible LysR system and the operons regulated by LysR are widely distributed across a broad range of microorganisms.

\section{Molecular modeling and docking}

Wek et al. reported that LysR-type protein binds to operator sites and directs a conformational change in the structure of the promoter-operator DNA complex [21]. When bound by inducer molecules, LysR protein enhances the recruitment of RNA polymerase to the promoter without affecting the occupancy of the LysR protein at the operator site. However, the ligand-associated conformational changes in LysR protein and their effect on the activity of LysR have not been fully elucidated. For detailed understanding of the structural and functional characteristics, protein modeling was performed for C4-LysR and its interaction with 3-HP (protein-ligand interaction). Because the crystal structure of C4-LysR is not available, multiple template threading was employed

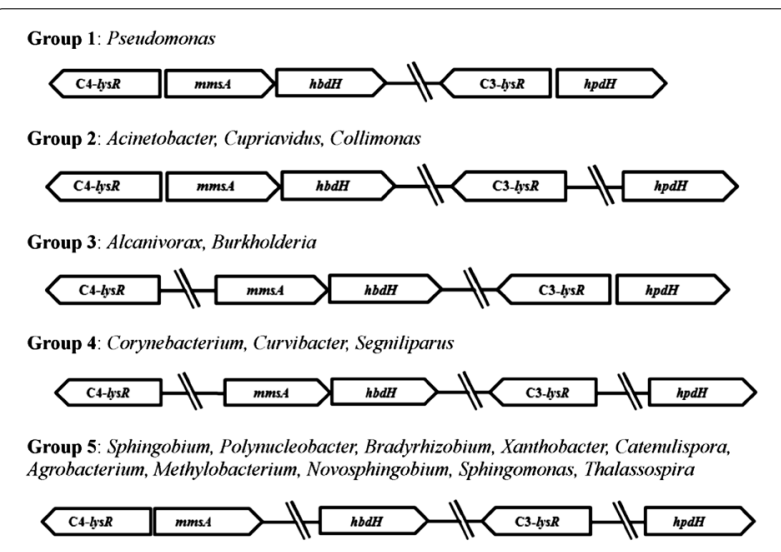

Group 6: Alicyclophihus, Alteromonas, Kitasatospora, Paracoccus, Simiduia, Sphingopyxis

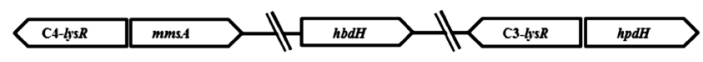

Group 7: Achromobacter, Acidovorax, Alcaligenes, Halomonas, Azospirillum, Chromobacterium, Comamonas

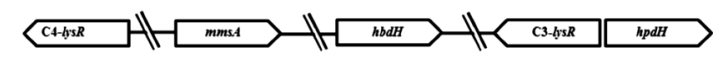

Group 8: Verminephrobacter, Janthinobacterium, Idiomarina, Glaciecola, Gordonia, Delftia, Pseudogulbenkiania, Bordetella

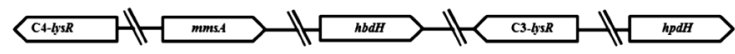

Group 9: Phenylobacterium, Psychrobacter, Shewanella, Vibrio, Xanthomonas, Stenotrophomonas, Caulobacter, Hahella, Kutzneria, Rhodomicrobium, Pseudoxanthomonas, photobacterium

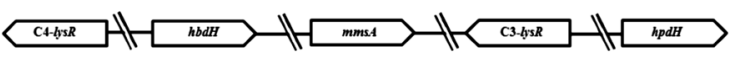

Group 10: Methylocystis, Tatlockia

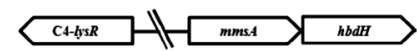

Group 11: Sinorhizobium, Beijerinckia, Aeromonas, Castellamiella, Amycolatopsis

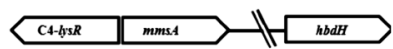

Group 12: Oceanimonas, Rastolnia, Pseudoalteromonas, Ferrimonas, Anaeromyxobacter

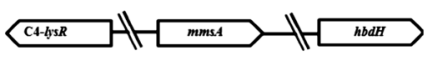

Group 13: Azotobacter

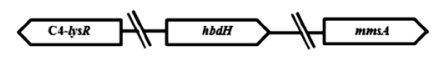

Group 14: Parvibaculum, Hirschia

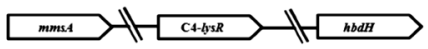

Fig. 4 Gene organization of C3 and C4 operons involved in 3-HP degradation pathway in various microbial genera

(see "Methods"). The predicted model was evaluated using the RAMPAGE tool, by calculating the main-chain RMSD (Root Mean Square Deviation) with reference to its template structure (3SZP) and their amino acid distribution.

Among the various docking poses generated, the best one showed the lowest Glide docking score (empirical scoring function) of $-5.01 \mathrm{kcal} / \mathrm{mol}$ with three hydrogen bonds and a hydrophobic contact, which indicated the 


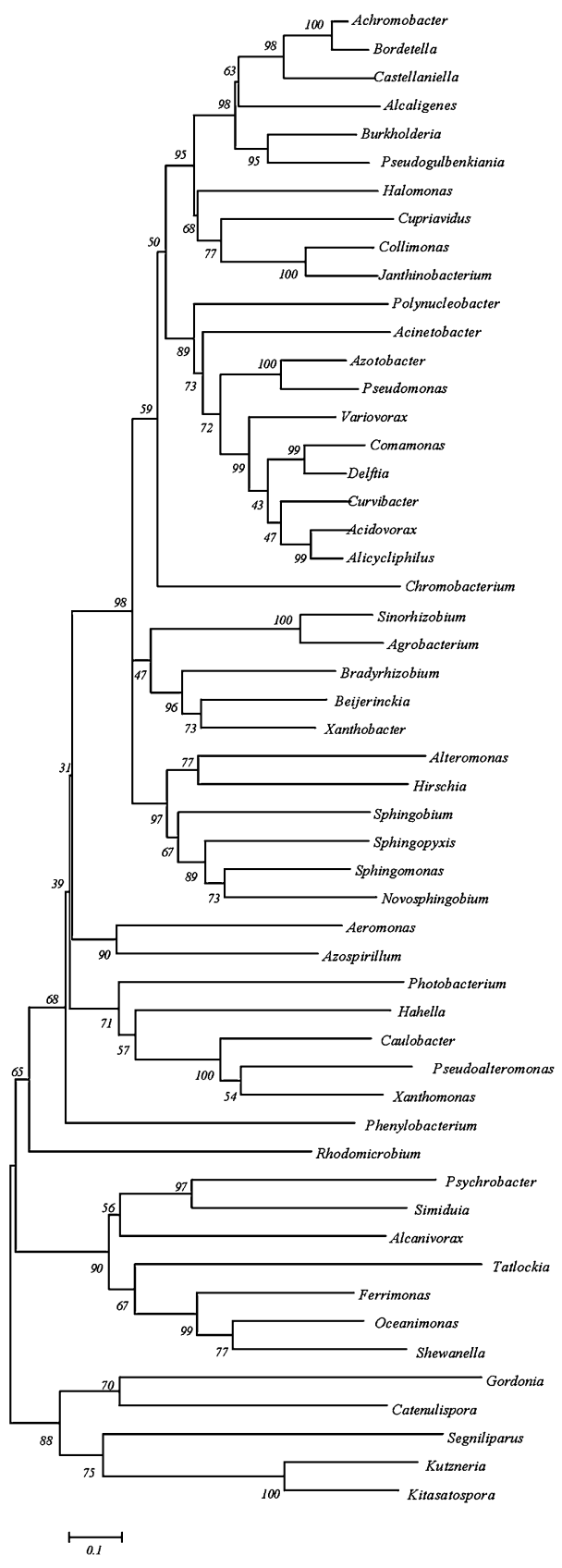

Fig. 5 Evolutionary relationships of C4-LysR in various microbial genera. The location of Pseudomonas in the tree is marked. The numbers on the tree branches represent the measure of statistical confidence in each branch of the tree; genera with confidence levels below 30 , generally indicative of lower confidence, were eliminated

high reliability of the simulation [22]. Several intermolecular interactions were identified between C4-LysR and the 3-HP molecule: three amino acid residues (ARG94, LYS96 and GLU137) present in the substrate-binding domain showed hydrogen bonding with 3-HP, while
Table 1 Bacterial strains used in this study and their 3-HP degradation

\begin{tabular}{lll}
\hline Strain & Source & 3-HP degraded (mM) \\
\hline Pseudomonas denitrificans & KCCM, Korea & 20.5 \\
\multicolumn{1}{c}{ ATCC13867 } & & \\
Pseudomonas knackmussii B13 & DSM, Germany & 7.4 \\
Pseudomonas protegens CHA0 & DSM, Germany & 25.2 \\
Pseudomonas fluorescens A506 & ATCC, America & 24.4 \\
Alicycliphilus denitrificans & DSM, Germany & 20.6 \\
Pseudogulbenkiania sp. & DSM, Germany & 7.4 \\
Collimonas arenae & DSM, Germany & 16.1 \\
Acidovorax sp. & DSM, Germany & 16.6 \\
Achromobacter xylosoxidans & KCCM, Korea & 18.4 \\
Acidovorax avenae subsp. citrulli & KCTC, Korea & 20.4 \\
Variovorax paradoxus & KCTC, Korea & 9.9 \\
\hline
\end{tabular}

a The 3-HP degradation was measured under non-growing conditions and the amount was calculated between 0 and $24 \mathrm{~h}$

ARG24 located in the helix-turn-helix domain, exhibited a hydrophobic interaction (Fig. 3). The steric hindrance caused by the binding of 3-HP to the active site of C4-LysR might be responsible for protein dimerization and DNA relaxation. Thus, increasing the RNA polymerase (RNAP) binding at the promoter site would effectively activate transcription of the target genes.

\section{Resting-cell degradation of 3-HP and 3-HP-dependent transcription activation of $\mathrm{C} 3$ and $\mathrm{C} 4$ operons in selected microorganisms}

As in the case of $P$. denitrificans, the genes in the C3 and C4 operons in the microorganisms presented in Fig. 4 were expected to get involved in 3-HP degradation and/ or assimilation. To confirm this, 3-HP degradation under non-growing conditions was examined for 10 selected microorganisms (Table 1). Cells were cultured in nutrient media containing $25 \mathrm{mM} 3-\mathrm{HP}$ to mid-log period, harvested by centrifugation, and resuspended in $100 \mathrm{mM}$ potassium phosphate buffer ( $\mathrm{pH} 7.0)$ containing $25 \mathrm{mM}$ 3-HP. After $24 \mathrm{~h}$ incubation at $30{ }^{\circ} \mathrm{C}$, the amount of degraded 3-HP was determined. All 10 of the microorganisms degraded 3-HP, though the amounts varied. Four strains, Pseudomonas protegens CHA0, Pseudomonas fluorescence A506, Alicycliphilus denitrificans and A. avenae subsp. citrulli, showed high degradation yields similar to that of $P$. denitrificans. In contrast, Pseudomonas knackmussii B13, Pseudogulbenkiania sp. and Variovorax paradoxus exhibited up to $50 \%$ lower degradation yields compared with $P$. denitrificans.

The transcription levels of the 3-HP catabolic genes ( $h p d H, h b d H, m m s A$ ) in the 10 microorganisms also were determined, after growing them in the absence or presence of $25 \mathrm{mM} 3-\mathrm{HP}$ (Table 2). Six showed improved 
Table 2 Fold increases of mRNA expression levels of $h p d H$, $m m s A$ and $h p d H$ by induction of 3-HP in various microorganisms

\begin{tabular}{llll}
\hline Strain & \multicolumn{3}{l}{ Fold increase } \\
\cline { 2 - 4 } & hpdH & mms & hbdH \\
\hline Pseudomonas denitrificans ATCC13867 & 47.0 & 142.4 & 140.9 \\
Pseudomonas knackmussii B13 & 10.4 & 3.9 & 4.2 \\
Pseudomonas protegens CHA0 & - & - & - \\
Pseudomonas fluorescens A506 & 24.6 & 9.1 & 17.8 \\
Alicycliphilus denitrificans & 6.3 & 37.3 & 20.2 \\
Pseudogulbenkiania sp. & 1.6 & 2.4 & 100.6 \\
Collimonas arenae & $\mathrm{ND}$ & $\mathrm{ND}$ & $\mathrm{ND}$ \\
Acidovorax sp. & 0.7 & 6.8 & 5.9 \\
Achromobacter xylosoxidans & $\mathrm{ND}$ & $\mathrm{ND}$ & $\mathrm{ND}$ \\
Acidovorax avenae subsp. citrulli & 7.7 & 33.1 & 14.0 \\
Variovorax paradoxus & $\mathrm{ND}$ & $\mathrm{ND}$ & $\mathrm{ND}$ \\
\hline
\end{tabular}

ND not detectable

a Failed to isolate RNA

transcription for the three 3-HP catabolic genes, with the exception of Acidovorax sp. for $h p d H$. As in the case of $P$. denitrificans, transcription of $h p d H$ was lower than that of $m m s A$ or $h b d H$, indicating that the promoter strength of the former is weaker than the others. In three of the microorganisms (Collimonas arenae, Achromobacter xylosoxidans, V. paradoxus), the mRNA of the 3-HP catabolic genes was not amplified at all, which suggested that the degenerate primer sequences, designed based on NCBI database, did not match the original gene sequence (Additional file 3: Table S3). With $P$. protegens CHA0, total RNA could not be isolated using standard RNA isolation procedures (see "Methods"). It should be noted that the fold increases in transcription of three genes in the six microorganisms (P. knackmussii B13, P. fluorescence A506, A. denitrificans, Pseudogulbenkiania sp., Acidovorax sp. and $A$. avenae subsp. citrulli) are generally lower compared to those of $P$. denitrificans (Table 2). In $P$. denitrificans, the fold increase was in the range of 47-142, while that in the other six microorganisms was $<37$. The reason for this difference is not clear, though we suspect that culture conditions, including medium composition, could have affected expressions of C3- and C4-LysR and the catabolic genes under their control. Nevertheless, the enhanced transcription of the putative 3-HP catabolic genes, along with the resting-cell 3-HP degradation, strongly suggests that the 10 microorganisms tested (Table 1) and, probably, most of other microorganisms listed in Additional files 1 and 2: Tables S1 and S2 have 3-HP-inducible C3 and/or C4 operons similar to those of $P$. denitrificans. Further in vivo and in vitro studies to elucidate the detailed mechanism of 3-HPinducible systems are in progress.

\section{Conclusions}

Unique transcriptional activator proteins and promoters that respond to 3-HP were studied. In the presence of 3-HP, two different LysR-family transcriptional regulators, designated C3- and C4-LysR, respectively, were found to stimulate the transcription of the catabolic genes $h p d H, h b d H$ and/or mmsA involved in 3-HP degradation. The inducible systems were common to many microorganisms: more than 53 genera, according to the genome sequence databases. The present molecular modeling and docking studies suggested that, in C4-LysR, the four amino acid residues ARG94, LYS96, GLU137 and ARG24 interact with 3-HP and activate the LysR regulator protein. The 3-HP-inducible systems promise to be of a great utility to the development of gene expression systems that are regulated by $3-\mathrm{HP}$.

\section{Methods}

Materials

Pseudomonas denitrificans ATCC 13867 and Pseudomonas fluorescens A506 were purchased from ATCC (America). A. xylosoxidans were obtained from KCCM (Korea). A. avenae sub sp. citrulli and V. paradoxus were obtained from KCTC (Korea). P. knackmussii B13, P. protegens, A. denitrificans, Pseudogulbenkiania sp., C. arenae and Acidovorax sp. were acquired from DSM (Germany). The primers were synthesized by Cosmo Genetech Co. Ltd (Seoul, Korea). 3-HP was purchased from Tokyo Kasei Kogyo Co. Ltd., Tokyo, Japan (TCI America, Portland, OR). Yeast extract (Cat. 212750), tryptone (Cat. 211705), trypticase soy broth (Cat. 211768), and peptone (Cat. 211921) were supplied by Difco (Becton-Dickinson; Franklin Lakes, NJ). Unless indicated otherwise, all of the other chemicals and enzymes were purchased from Sigma-Aldrich (St. Louis, MO).

\section{Resting-cell degradation of 3-HP}

Non-growing cell experiments were performed to examine 3-HP degradation by some of the bacteria strains listed in Table 1. Active cells were prepared by growing the strains each in its own specified enriched nutrient medium using $250 \mathrm{~mL}$ Erlenmeyer flasks with $50 \mathrm{~mL}$ working volume under aerobic conditions with $200 \mathrm{rpm}$ agitation speed in an orbital incubator shaker. The nutrient medium for the $P$. fluorescence strain contains the following components per liter: peptone, $20 \mathrm{~g}$; glycerol, $10 \mathrm{~mL} ; \mathrm{K}_{2} \mathrm{HPO}_{4}, 1.5 \mathrm{~g} ; \mathrm{MgSO}_{4} \cdot 7 \mathrm{H}_{2} \mathrm{O}, 1.5 \mathrm{~g} ; 3-\mathrm{HP}$, $25 \mathrm{mmol}$. For the P. knackmussii, P. protegens, Pseudogulbenkiania sp., A. xylosoxidans, V. paradoxus, Acidovorax avenae subsp. citrulli, $C$. arenae and $A$. denitrificans 
strains, the nutrient medium contains the following components per liter: peptone, 5 g; beef extract $3 \mathrm{~g}$; 3-HP, $25 \mathrm{mmol}$. For the Acidovarax sp. strain, trypticase soy broth containing $25 \mathrm{mmol} / \mathrm{L}$ was used. The cultures were conducted at $30{ }^{\circ} \mathrm{C}$, obtaining $\sim 1-1.5 \mathrm{OD}_{600}$. The cells were then harvested and centrifuged at $5000 \mathrm{rpm}$ for $10 \mathrm{~min}$. The resultant pellet was washed with $100 \mathrm{mM}$ potassium phosphate buffer ( $\mathrm{pH} 7.0)$ and resuspended in the same buffer supplemented with $25 \pm 2 \mathrm{mmol} / \mathrm{L} 3-\mathrm{HP}$. The cells were then harvested and centrifuged as above noted, subjecting them to non-growing 3-HP degradation experimentation. After $24 \mathrm{~h}$ cultivation, the samples were withdrawn to determine the 3-HP concentrations.

\section{RNA extraction and Real-time PCR}

The P. denitrificans ATCC 13867 strain was grown in M9 minimal medium containing $5 \mathrm{~g} / \mathrm{L}$ sodium gluconate; the other microorganism strains listed in Table 1 were grown in a specified nutrient medium. The cells were cultivated under the aerobic condition at 37 or $30^{\circ} \mathrm{C}$ and $200 \mathrm{rpm}$ in an orbital incubator shaker. 3-HP at $0 / 25 \mathrm{mM}$ was supplemented at $\mathrm{OD}_{600}$ of $\sim 0.4-0.5$. After a further $2 \mathrm{~h}$ cultivation, approximately $5 \times 10^{8}$ cells were collected and centrifuged at $5000 \times g$ for $10 \mathrm{~min}$. The cell pellets were immediately resuspended in $500 \mu \mathrm{L}$ of RNAlater solution (Ambion, UK). RNA was extracted using a total RNA isolation kit (Macherey-Nagel, Germany). One microgram of total RNA was employed to synthesize the first-strand cDNA in a $20 \mu \mathrm{L}$ reaction using the SuperScript III firststrand synthesis system (Invitrogen, USA). A real-time PCR analysis was performed, according to the SYBR green method, in a $20 \mu \mathrm{L}$ reaction volume using the StepOne Real-Time PCR system (Applied Biosystems, USA). The PCR efficiencies of all of the primers were experimentally determined and found to be suitable for reliable copy-number quantification. The relative quantification for each of the mRNA levels was calculated using the $\Delta \Delta C_{\mathrm{t}}$ method as described previously [23]. All of the assays were performed in duplicate, and a template-less reaction template was used as a negative control.

\section{Phylogenetic tree}

The evolutionary history was inferred using the Neighbor-Joining method [24], which proceeds as follows: a bootstrap consensus tree inferred from 1000 replicates is taken to represent the evolutionary history of the taxa analyzed [25]; branches corresponding to partitions reproduced in less than $50 \%$ of the bootstrap replicates are collapsed; the percentages of replicate trees in the associated taxa clustered together in the bootstrap test (1000 replicates) are shown next to the branches; the tree is drawn to scale, with branch lengths (in the same units as those of the evolutionary distances) used to infer the phylogenetic tree. The evolutionary distances were computed, using the Poisson correction method [26], in units of the number of amino acid substitutions per site. Evolutionary analyses were conducted in MEGA5 [27].

\section{Protein modeling and docking of C4-LysR}

A model three-dimensional (3D) C4-LysR structure was created by protein fold modeling using the MUSTER (MUlti-Source ThreadER) program [28]. The protein model with the lowest level probability density function (PDF) energy was selected. The prepared protein model was energy minimized by the OPLS 2005 force field. Further, the model, thus refined, was validated using ProCheck and Ramachandran plot (RAMPAGE tool; http:// raven.bioc.cam.ac.uk/rampage.php).

A molecular docking study was carried out to examine the binding interaction between the modeled C4-LysR protein and 3-HP. The active-site regions in C4-LysR for 3-HP binding were predicted, using the $\mathrm{COACH}$ tool, according to the highest $\mathrm{C}$-scores and cluster sizes of 0.05 and 9.0, respectively. The validated model and predicted active-site residues were used to perform docking studies, using the Maestro program from the SCHRODINGER $^{\mathrm{TM}}$ v10.1 software package to run the docking protocol. Briefly, the target protein (C4-LysR) and the ligand (3-HP) were prepared and processed using Protein Preparation Wizard and LigPrep Wizard in the Schrodinger graphical user interface MAESTRO (version 10.1). Bond orders were assigned to the ligand, and hydrogen bonds consistent with the physiological $\mathrm{pH}$ (7.0) were added to the receptor. The initial ligand conformations were obtained by a Monte Carlo conformational search. Using the Receptor Grid Generation tool, a receptor grid box (scaling factor: 1.0; partial charge cutoff: $0.25 \AA$ ) was generated around the active-site residues predicted by the $\mathrm{COACH}$ tool. Ligand docking was performed using XP (extra precision) predefined docking settings and flexible ligand sampling within the grid box. Finally, the docked poses were visualized using the Maestro 10.1 graphical user interface.

\section{Analytical methods}

The cell concentration was determined in a $10-\mathrm{mm}$-pathlength cuvette using a double-beam spectrophotometer (Lambda 20, Perkin-Elmer, Norwalk, CT). The concentrations of 3-HP were determined by HPLC using a slightly modified version of the method described elsewhere [29]. Briefly, the obtained by $10 \mathrm{~min}$ centrifugation of the culture samples at $10,000 \times g$ was filtered through a Tuffryn-membrane (Acrodisc; Pall Life Sciences, Port Washington, NY) and eluted through a $300 \mathrm{~mm} \times 7.8 \mathrm{~mm}$ Aminex HPX-87H (Bio-Rad, USA) column at $65^{\circ} \mathrm{C}$ using $2.5 \mathrm{mmol} / \mathrm{L} \mathrm{H}_{2} \mathrm{SO}_{4}$ as the mobile phase. 


\section{Additional files}

Additional file 1: Table S1. Comparison of sequence homologies between C3-operon proteins from P. denitrificans and other organisms.

Additional file 2: Table S2. Comparison of sequence homologies between C4-operon proteins from $P$. denitrificans and other organisms.

Additional file 3: Table S3. Primers used for RT PCR in this study.

\section{Abbreviations}

$\mathrm{NAD}^{+}$: oxidized form of nicotinamide adenine dinucleotide; RT-PCR: real-time polymerase chain reaction; $C_{t}$ : cycle threshold.

\section{Authors' contributions}

SZ, SKA and SP designed the research. SZ, SKA and TTN performed the experiment. ES helped with strain ordering and experimental analysis. SZ, SKA and SP analyzed the data and wrote the manuscript. All authors read and approved the manuscript.

\section{Author details}

1 School of Chemical and Biomolecular Engineering, Pusan National University, San 30 Jangeon-dong, Geumjeong-gu, Busan 609-735, Republic of Korea.

${ }^{2}$ Department of Biochemical Engineering, College of Pharmaceutical and Life Sciences, Changzhou University, Changzhou 213164, China.

\section{Acknowledgements}

This study was supported by the Korean Ministry of Science, ICT and Future Planning through the Advanced Biomass R \& D Center (ABC; Grant No. 20100029799), KAIST, Korea. This study was also supported by the "2015 Post-Doc. Development Program" of Pusan National University (for S. Zhou) and the BK 21 Plus Program for Advanced Chemical Technology at Pusan National University (for S. K. Ainala and T. T. Nguyen).

\section{Competing interests}

The authors declare that they have no competing interests.

Received: 29 July 2015 Accepted: 2 October 2015

Published online: 20 October 2015

\section{References}

1. Paster M, Pellegrino JL, Carole TM. Industrial bioproducts: today and tomorrow. US DOE report. 2003. http://www.brdisolutions.com/pdfs/ BioProductsOpportunitiesReportFinal.pdf.

2. Pina CD, Falletta E, Rossi M. A green approach to chemical building blocks. The case of 3-hydroxypropanoic acid. Green Chem. 2011;13:1624-32.

3. Zhang D, Hillmyer MA, Tolman WB. A New Synthetic route to poly[3hydroxypropionic acid] (P[3-HP]): ring-opening polymerization of 3-HP macrocyclic esters. Macromolecules. 2004;37:8198-200.

4. Ashok S, Sankaranarayanan M, Ko Y, Jae KE, Aniala SK, Vinod K, et al. Production of 3-hydroxypropionic acid from glycerol by recombinant Klebsiella pneumoniae $\Delta d h a T \Delta y q h D$ which can produce vitamin $B_{12}$ naturally. Biotechnol Bioeng. 2012;110:511-24.

5. Kim K, Kim SK, Park YC, Seo JH. Enhanced production of 3-hydroxypropionic acid from glycerol by modulation of glycerol metabolism in recombinant Escherichia coli. Bioresour Technol. 2014;156:170-5.

6. Rathnasingh C, Raj SM, Jo JE, Park S. Development and evaluation of efficient recombinant Escherichia coli strains for the production of 3-hydroxypropionic acid from glycerol. Biotechnol Bioeng. 2009;104:729-39.

7. Zhou S, Catherine C, Rathnasingh C, Somasundar A, Park S. Production of 3-hydroxypropionic acid from glycerol by recombinant Pseudomonas denitrificans. Biotechnol Bioeng. 2013;110:3177-87.
8. Zhou S, Ashok S, Ko Y, Kim DM, Park S. Development of a deletion mutant of Pseudomonas denitrificans that does not degrade 3-hydroxypropionic acid. Appl Microbiol Biotechnol. 2014;98:4389-98.

9. Sankaranarayanan M, Ashok S, Park S. Production of 3-hydroxypropionic acid from glycerol by acid tolerant Escherichia coli. J Ind Microbiol Biotechnol. 2014;41:1039-50.

10. Toraya $T$. Radical catalysis of $B_{12}$ enzymes: structure, mechanism, inactivation, and reactivation of diol and glycerol dehydratases. Cell Mol Life Sci. 2000:57:106-27.

11. Yamanishi M, Kinoshita K, Fukuoka M, Saito T, Tanokuchi A, Ikeda Y, et al. Redesign of coenzyme $B_{12}$ dependent diol dehydratase to be resistant to the mechanism-based inactivation by glycerol and act on longer chain 1,2-diols. FEBS J. 2012:279:793-804.

12. Franken B, Eggert T, Jaeger KE, Pohl M. Mechanism of acetaldehydeinduced deactivation of microbial lipases. BMC Biochem. 2011;12:1-10.

13. Di Lorenzo M, Hidalgo A, Molina R, Hermoso JA, Pirozzi D, Bornscheuer UT. Enhancement of the stability of a prolipase from Rhizopus oryzae toward aldehydes by saturation mutagenesis. Appl Environ Microbiol. 2007;73:7291-9.

14. Weber HK, Stecher H, Faber K. Sensitivity of microbial lipases to acetaldehyde formed by acyl-transfer reactions from vinyl esters. Biotechnol Lett. 1995; 17:803-8.

15. Weber HK, Zuegg J, Faber K, Pleiss J. Molecular reasons for lipase-sensitivity against acetaldehyde. J Mol Catal B Enzym. 1997;3:131-8.

16. Jennewein S, Schurmann M, Wolberg M, Hilker I, Luiten R, Wubbolts M, et al. Directed evolution of an industrial biocatalyst: 2-deoxy-D-ribose5-phosphate aldolase. Biotechnol J. 2006;1:537-48.

17. Ainala SK, Somasundar A, Park S. Complete genome sequence of Pseudomonas denitrificans ATCC13867. Genome Announc. 2013;1:e00257-13.

18. Lee P, Raj SM, Zhou S, Ashok S, Edwardraja S, Park S. 3-Hydroxyisobutyrate dehydrogenase-I from Pseudomonas denitrificans ATCC13867 degrades 3-hydroxyprpionic acid. Biotechnol Bioprocess Eng. 2014;19:1-7.

19. Zhou S, Mohan Raj S, Ashok S, Edwardraja S, Lee SG, Park S. Cloning, expression and characterization of 3-hydroxyisobutyrate dehydrogenase from Pseudomonas denitrificans ATCC 13867. PLoS One. 2013;8:e62666.

20. Moddocks SE, Oyston PCF. Structure and function of the LysRtype transcriptional regulator (LTTR) family proteins. Microbiology. 2008;154:3609-23.

21. Wek RC, Hatfield GW. Transcriptional activation at adjacent operators in the divergent-overlapping ilvY and ilvC promoters of Escherichia coli. J Mol Biol. 1998;203:643-63.

22. Shukla S, Datt M, Chance MR, Gupta S. Apigenin suppresses IKK activation and downstream signaling leading to prostate cancer inhibition. Cancer Res. 2013:73:1-3660.

23. Yim SH, Kim TM, Hu HJ, Kim JH, Kim BJ, Lee JY, et al. Copy number variations in East-Asian population and their evolutionary and functional implications. Hum Mol Genet. 2010;19:1001-8.

24. Saitou N, Nei M. The neighbor-joining method: a new method for reconstructing phylogenetic trees. Mol Biol Evol. 1987;4:406-25.

25. Felsenstein J. Confidence limits on phylogenies: an approach using the bootstrap. Evolution. 1985;39:783-91.

26. Zuckerkandl E, Pauling L. Evolutionary divergence and convergence in proteins. In: Bryson V, Vogel HJ, editors. Evolving Genes and Proteins. New York: Academic Press; 1965. p. 97-166.

27. Tamura K, Peterson D, Peterson N, Stecher G, Nei M, Kumar S. MEGA5: molecular evolutionary genetics analysis using maximum likelihood, evolutionary distance, and maximum parsimony methods. Mol Boil Evol. 2011;28:2731-9.

28. Wu S, Zhang Y. MUSTER: improving protein sequence profile-profile alignments by using multiple sources of structure information. Proteins. 2008;72:547-56.

29. Raj SM, Rathnasingh C, Jo JE, Park S. Production of 3-hydroxypropionic acid from glycerol by a novel recombinant Escherichia coli BL21 strain. Process Biochem. 2008:43:1440-6. 